\title{
CXCR4 signalling, metastasis and immunotherapy: zebrafish xenograft model as translational tool for anti-cancer discovery
}

\author{
Claudia Tulotta, B. Ewa Snaar-Jagalska \\ IBL Animal Sciences \& Health, Institute of Biology Leiden, Leiden University, Leiden, CC 2333, the Netherlands.
}

Correspondence to: Dr. B. Ewa Snaar-Jagalska, IBL Animal Sciences \& Health, Institute of Biology Leiden, Leiden University, Einsteinweg 55, Leiden, CC 2333, the Netherlands. E-mail: b.e.snaar-jagalska@biology.leidenuniv.nl

How to cite this article: Tulotta C, Snaar-Jagalska BE. CXCR4 signalling, metastasis and immunotherapy: zebrafish xenograft model as translational tool for anti-cancer discovery. J Cancer Metastasis Treat 2019;5:74.

http://dx.doi.org/10.20517/2394-4722.2019.022

Received: 14 Aug 2019 First Decision: 20 Sep 2019 Revised: 18 Oct 2019 Accepted: 31 Oct 2019 Published: 8 Nov 2019

Science Editor: Pravin D. Potdar Copy Editor: Cai-Hong Wang Production Editor: Jing Yu

\begin{abstract}
Cell-to-cell communication guarantees homeostasis in a multi-cellular organism. Cancer-to-microenvironment communication sustains malignant growth and dissemination. Whereas the accumulation of mutations is at the origin of malignant cell transformation and neoplasia onset, the interaction between cancer and the surrounding stroma, specifically immune cells, influences the balance between tumour regression and tumour progression. To study how the interaction between cancer and stromal cells is disadvantageous or beneficial for tumour progression, the use of a transparent in vivo model bears important research potentials. Zebrafish has been increasingly used as animal model to study tumour biology. The use of transparent zebrafish embryos, with fluorescent endothelial and immune cells, allows the visualization of cell-to-cell interaction, among host cells themselves and between zebrafish stroma and implanted human cancer cells. Here, we summarise our findings on the role of CXCR4 signalling in tumour progression, considering its signature both on cell autonomous and host dependent mechanisms. Finally, we address the translational impact of targeting CXCR4 signalling in cancer and the tumour microenvironment for the treatment of metastatic cancer.
\end{abstract}

Keywords: CXCR4, cancer, metastasis, neutrophils, zebrafish, immunotherapy 


\section{THE TUMOUR MICROENVIRONMENT}

Tumours are in constant interaction with the surrounding microenvironment. The tumour microenvironment consists of stromal cells such as cancer-associated fibroblasts (CAFs), endothelial cells, mesenchymal stem cells (MSCs), tumour-associated macrophages (TAMs) and neutrophils (TANs), adaptive immune cells and extracellular matrix $(\mathrm{ECM})^{[1]}$. The interaction between cancer and stroma cells results in either tumour promoting or inhibiting effects and the tumour microenvironment differentially contributes to the efficacy of cancer therapies ${ }^{[2]}$. Tumour cells engage cells from the microenvironment, either educating resident stromal cells or inducing the recruitment of distal ones to further support malignant growth, motility and dissemination. Along with the angiogenic switch, where endothelial cells are educated by malignant cells to form new vasculature to provide oxygen and nutrients, the immunosuppressive switch phenomenon takes place: the polarization from pro-inflammatory to antiinflammatory neutrophils and macrophages ( $\mathrm{N}_{1}$ to $\mathrm{N} 2$ and $\mathrm{M} 1$ to $\mathrm{M} 2$ ), where the sub-type 2 associates with a tumour-promoting function, links to immunosuppression, characterized by reduced cytotoxic $\mathrm{T}$ cell and enhanced $\mathrm{T}$ regulatory (Treg) and myeloid-derived suppressor (MDSCs) cell infiltration ${ }^{[3]}$. Interestingly, the cooperation between different subsets of leukocytes and its role in cancer metastases has been recently reported ${ }^{[4]}$. The plasticity phenomenon in the microenvironment has been described also for fibroblasts, which respond to a neoplastic lesion in a similar fashion as to a never healing wound ${ }^{[3]}$. The interaction between tumour and the microenvironment is controlled by a plethora of signalling molecules, such as chemokines, and their complex networking in cancer requires further understanding to inhibit tumour development.

\section{CXCL12-CXCR4 AXIS IN CANCER AND THE TUMOUR MICROENVIRONMENT}

Chemokines are chemotactic cytokines that guide directional cell migration in development and disease and more than 50 chemokine ligands and 18 chemokine receptors have been described in Homo sapiens ${ }^{[5]}$. Chemokines are classified into four classes, depending on the presence and position of the conserved cysteine residues (CXC, $\mathrm{CC},(\mathrm{X}) \mathrm{C}$ and $\mathrm{CX} 3 \mathrm{C}$ ) at the $\mathrm{N}$-terminus, involved in the formation of disulphide bonds between the first and third or second and fourth cysteines ${ }^{[6]}$. The chemokines belonging to the CXC subgroup are further classified into angiogenic ELR+ and angiostatic ELR-, whether they are positive or negative for the Glu-Leu-Arg (ELR) motif at the N-terminus ${ }^{[7,8]}$. Chemokine ligands can bind multiple chemokine receptors, which possibly work in concert to control signalling activation and inhibition ${ }^{[8]}$.

CXCR4 is a seven-transmembrane, chemokine, G-protein coupled receptor. The chemokine CXCL12 binds both CXCR4 and CXCR7 receptors in order to guide a directional and collective migration of cell primordia, during the formation of sensory organs in zebrafish ${ }^{[9-11]}$. CXCL12 binding to CXCR4 induces the dissociation of the G protein $\alpha \beta \gamma$ trimer and activation of PI3K/AKT/mTOR, MAPK, PKA and PLC/ $\mathrm{Ca}^{2+}$ pathways. Moreover, MAPK cascade activation and CXCR4 internalization occur via $\beta$-Arrestin, independently from G-proteins [Figure 1A]. In addition, CXCR4 can form homodimers, activating the JAK/STAT pathway and $\mathrm{Ca}^{2+}$ release from intracellular storage into the cytoplasm [Figure 1B]. CXCR4 can also form heterodimers with CXCR7. Whereas CXCR4 is internalized and degraded after CXCL12 binding, CXCR7 is internalized and recycled to the plasma membrane. Via $\beta$-Arrestin, CXCR7 has either CXCL12 scavenging functions or triggers MAPK signalling activation [Figure 1C]. CXCL12 signalling via CXCR4 and CXCR7 controls cell chemotaxis and migration as well as cell proliferation and survival ${ }^{[12,13]}$.

In cancer, malignant cells acquire higher CXCR4 levels, compared to normal tissues, and are found to preferentially metastasise in organs where CXCL12 is secreted, in line with the "seed and soil" theory ${ }^{[14]}$. Enhanced CXCR4 signalling has been identified in several malignancies such as gastrointestinal tumours ${ }^{[15]}$, melanoma ${ }^{[16]}$, basal cell carcinoma ${ }^{[17]}$, head and neck squamous cell carcinoma ${ }^{[18]}$, lung cancer ${ }^{[19]}$, breast $^{[20]}$ and ovarian ${ }^{[21]}$ tumours, renal cell carcinoma ${ }^{[22]}$, prostate cancer ${ }^{[23]}$, glioblastoma multiforme 


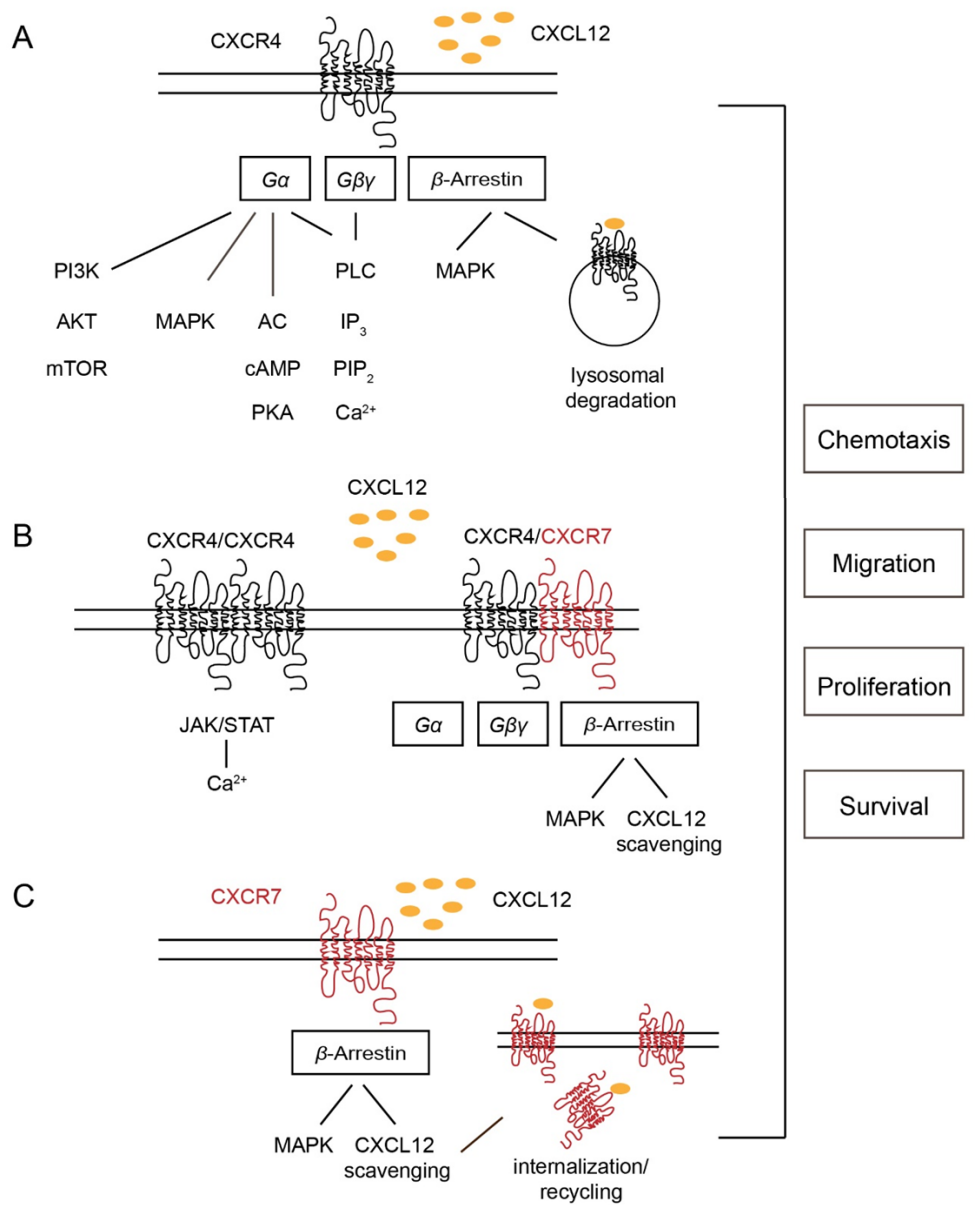

Figure 1. CXCL12-induced signalling via CXCR4 and CXCR7. (A) CXCL12 binds to CXCR4, inducing $G \alpha$ and $G \beta \gamma$ dissociation and activation of PI3K, MAPK, AC, and PLC signalling pathways. CXCL12 binding to CXCR4 activates $\beta$-Arrestin, leading to MAPK signalling pathway activation or receptor internalization. (B) CXCR4 can form homo- and hetero-dimers with CXCR7. (C) CXCL12 binding to CXCR7 induces, via $\beta$-Arrestin, MAPK signalling activation, or CXCL12 scavenging, through receptor internalisation and recycling to the plasma membrane. CXCL12-mediated signalling plays a role in cell chemotaxis, migration, proliferation and survival. PI3K: phosphatidylinositide 3-kinase; MAPK: mitogen-activated protein kinases; AC: adenyly cyclase; PLC: phospholipase C

$(\mathrm{GBM})^{[24]}$, Ewing sarcoma ${ }^{[25]}$ and leukemia ${ }^{[26]}$. Elevated CXCR4 levels result in increased cell proliferation, dedifferentiation, migration and metastatic spreading of tumour cells, cancer stem cell (CSC) maintenance and it has been associated with the development of tumour resistance towards conventional therapies, leading to poor patient prognosis ${ }^{[27]}$.

CXCR4 is expressed by both cancer cells and surrounding stromal cells [Figure 2]. The recruitment of stromal cells expressing CXCR4 can be guided by the secretion of CXCL12 by cancer cells themselves or other stromal cells, such as MSCs and CAFs ${ }^{[28]}$. Moreover, CXCL12 secreted by CAFs displays effects on tumour cells, enhancing invasive potential ${ }^{[29]}$ and functioning as a protective shield against $\mathrm{T}$ cells, boosting immune escaping mechanisms ${ }^{[30]}$. In this context, pharmacological inhibition of CXCR4, resulted in redistribution of $\mathrm{CD} 3+\mathrm{T}$ cells within the "cancer cell nest", as defined by the authors, causing reduced cancer cell growth and improved response to check-point inhibitors ${ }^{[31]}$. CXCR4 is involved in leukocyte trafficking, hematopoietic stem progenitor cells homing and neutrophil retention in the bone marrow during homeostasis, inflammation, infection and cancer ${ }^{[12,32-35]}$. Infiltration of CXCR4hi neutrophils associates with faster tumour growth and angiogenesis in IFN $\beta$ deficient mice, injected with melanoma and fibrosarcoma ${ }^{[36]}$. CXCR4hi macrophages have been identified in CXCL12-enriched tumour 


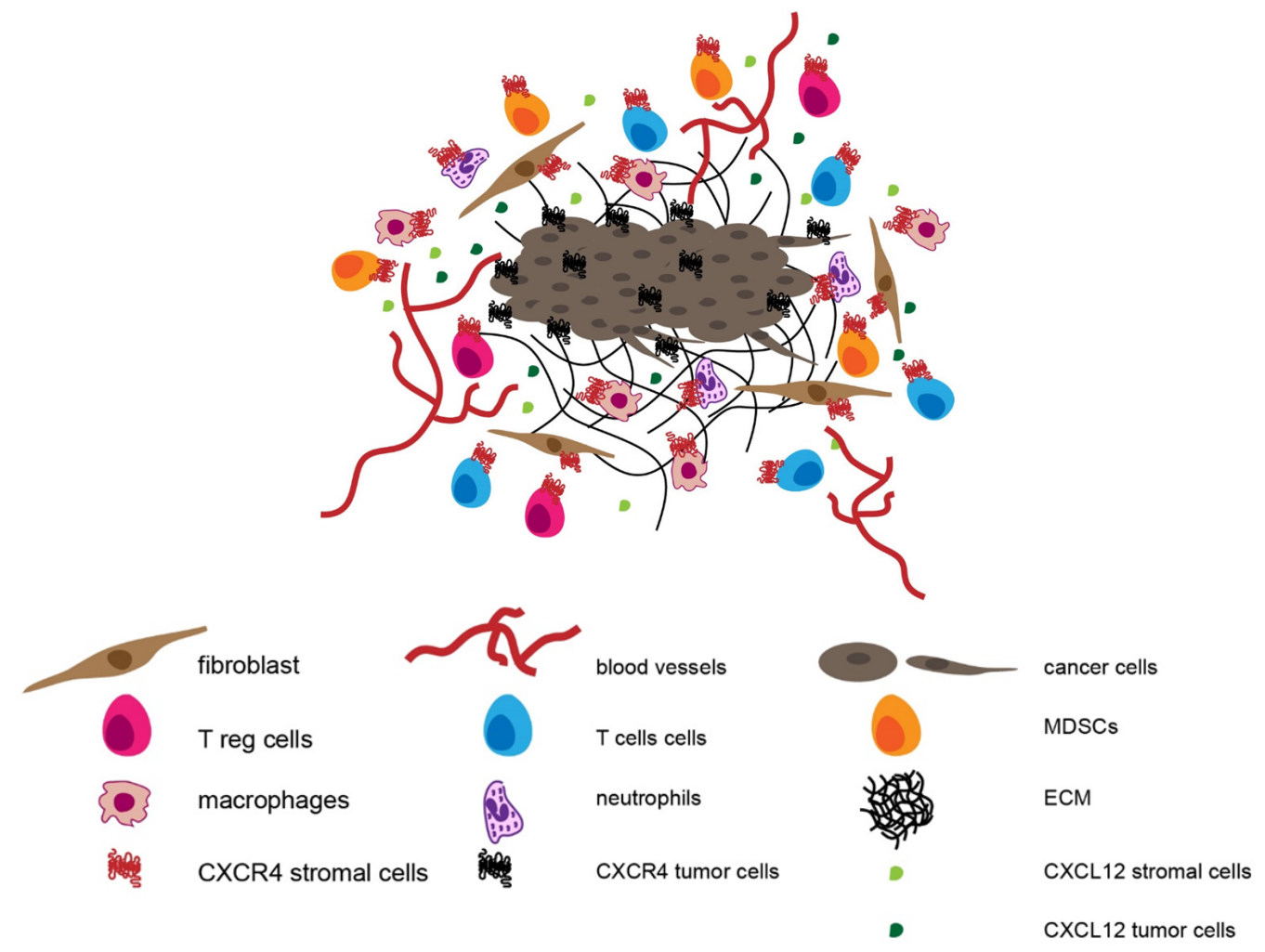

Figure 2. CXCR4 drives the interaction between cancer and stromal cells. The CXCR4-CXCL12 axis signals in a bi-directional fashion. CXCR4 is expressed by both tumour cells and cells that form the surrounding stroma [fibroblast, $T$ cells, $T$ reg cells, myeloid derived suppressors cells (MDSCs), macrophages and neutrophils], embedded in the extracellular matrix (ECM). The CXCR4 cognate ligand CXCL12 is secreted by both cancer cells and cells in the microenvironment

areas after chemotherapies and are suggested to display pro-angiogenic functions that drive tumourrelapse ${ }^{[37]}$. Moreover, CXCL12 expressing glioblastoma cells induce VEGF production and angiogenesis in microvessel enriched areas with high CXCR4 levels ${ }^{[38]}$. In addition, CXCR4-expressing peripheral blood monocytes respond to CXCL12-secreting multiple myeloma (MM) tumour cells and acquire M2 associated properties $^{[39]}$. Finally, the inhibition of CXCR4 signalling by oncolytic virotherapy limits the infiltration of Treg, decreasing immunosuppression ${ }^{[40]}$.

Considering the major and intricate role of this chemokine receptor in cancer, its targeting represents an important pharmacological approach that is currently under development, through the use of CXCR4 antagonists, antibodies and CXCL12 binding agents. Importantly, the role of the stromal CXCR4 signalling needs to be considered in drug treatments that target CXCR4 to inhibit cancer spreading.

In 2018, the Nobel prize in Physiology and Medicine was awarded to J.P. Allison and T. Honjo for the development of immune-checkpoint blockade ${ }^{[41]}$. This revolutionary discovery clearly underlines the wellknown pivotal role of the immune system in cancer. Inhibition of CXCR4 signalling has been found to improve the efficacy of immunotherapies in metastatic breast cancer, by alleviation of desmoplasia and increased $\mathrm{T}$ cell infiltration in preclinical in vivo models ${ }^{[42]}$.

Limiting cancer spreading by targeting CXCR4 signalling in the tumour microenvironment is a promising approach that requires further investigations to become an alternative therapeutic form of intervention. 


\section{ZEBRAFISH XENOGRAFT AS A MODEL TO STUDY CANCER}

Research performed in pre-clinical in vivo models is constantly under development to provide further insights into the communication between tumour and the surrounding microenvironment. Zebrafish (Danio rerio) is a tropical freshwater teleost, increasingly used to study a range of disease processes ${ }^{[43]}$ as well as being an excellent tool for the study of development. Several important advances in understanding of cancer and inflammation have arisen from studies in zebrafish ${ }^{[4-46]}$. The rapid and external development of transparent embryos ${ }^{[47]}$, availability of reporter lines with traceable fluorescent cells ${ }^{[48-50]}$, ease of genetic manipulation ${ }^{[51,52]}$ and pharmacological approaches ${ }^{[53]}$ make the zebrafish an excellent in vivo model to visualise single cell interactions in real time and to uncover the signalling mechanisms involved, on a whole organism level. Zebrafish is increasingly used as a model organism to study cancer ${ }^{[54]}$. There is high conservation of oncogenes and tumour-suppressor genes between zebrafish and human therefore data collected in zebrafish are relevant for humans ${ }^{[55]}$. The histology of zebrafish tumours has been shown to be highly similar to tumours found in human cancers ${ }^{[56]}$. Moreover, zebrafish is a valuable tool to study drug discovery in the context of cancer research ${ }^{[57,58]}$. Zebrafish larvae can absorb small molecular weight compounds from water, which is advantageous when screening for anti-cancer compounds ${ }^{[59]}$. The experimental costs are low and procedure are simple and fast. This accounts for the experimental increase in the use of zebrafish in drug discovery during the last two decades in a time- and cost- effective manner. For melanoma, a presently on-going phaseI/II clinical trial of Leflunomide combined with vemurafenib is the first to arise from initial screen in zebrafish. To study human cancer metastasis, our group generated a xenotransplantation model of experimental micrometastasis ${ }^{[60,6]}$. Human tumour cells engrafted into the blood circulation of 2-day-old zebrafish embryos induce angiogenesis and form micrometastasis sustained by neutrophils and macrophages, nearby hematopoietic sites ${ }^{[6]}$. In particular, tumour-induced angiogenesis, metastasis formation and relative chemical approaches to inhibit these processes have been studied using zebrafish as a xenotransplantation model, complementing current knowledge developed through the use of in vitro and other in vivo models ${ }^{[62]}$. Upon localised or haematogenous engraftment of cancer cells, zebrafish xenografts allow qualitative and quantitative assessment of tumour burden and tumour-microenvironment interaction, representing a powerful pre-clinical model to unravel cancer mechanisms and to develop new therapeutic strategies ${ }^{[61]}$. In particular, alongside murine models, the use of PDXs in zebrafish has the potential to be used in personalised medicine ${ }^{[63-66]}$, with the advantage of requiring less tumour material and shorter times for the monitoring of tumour development ${ }^{[57]}$. Several studies have shown that the combined use of zebrafish and murine models paves the way towards important insights to elucidate the biology of metastatic cancers and the development of new treatments ${ }^{[67-71]}$. Therefore, the zebrafish xenograft model bears the potential to elucidate crucial kinetics and key mechanisms that regulate tumour-microenvironment interaction and ultimately support tumour spreading.

\section{CELL-AUTONOMOUS CXCR4 SIGNALLING: THE CXCR4 ANTAGONIST IT1T IMPAIRS EARLY HUMAN METASTATIC EVENTS, IN A ZEBRAFISH XENOGRAFT MODEL WHERE THE INTERSPECIES CROSS-TALK TAKES PLACE}

Chemokines direct tumour and stromal cell bidirectional migration ${ }^{[72]}$. CXCR4 plays a physiological role in hematopoiesis ${ }^{[73,74]}$, leukocyte trafficking ${ }^{[75-77]}$, cell migration and embryo development ${ }^{[78]}$, as well as a pathological function in HIV pathogenesis ${ }^{[79]}$, WHIM syndrome ${ }^{[80]}$ and cancer $^{[81,82]}$. In addition to its cognate ligand CXCL12, CXCR4 can bind ubiquitin ${ }^{[83]}$, macrophage migration inhibitory factor ${ }^{[84-86]}$ and CXCL14 ${ }^{[87]}$. The CXCR4-CXCL12 signalling axis is known to play a critical function in cancer cell spreading, when tumour cells expressing high levels of CXCR4 communicate with CXCL12-secreting stromal cells of distant organs that function as metastatic and secondary growth "soils" 
A Functional Cell-autonomous and Host-dependent CXCR4

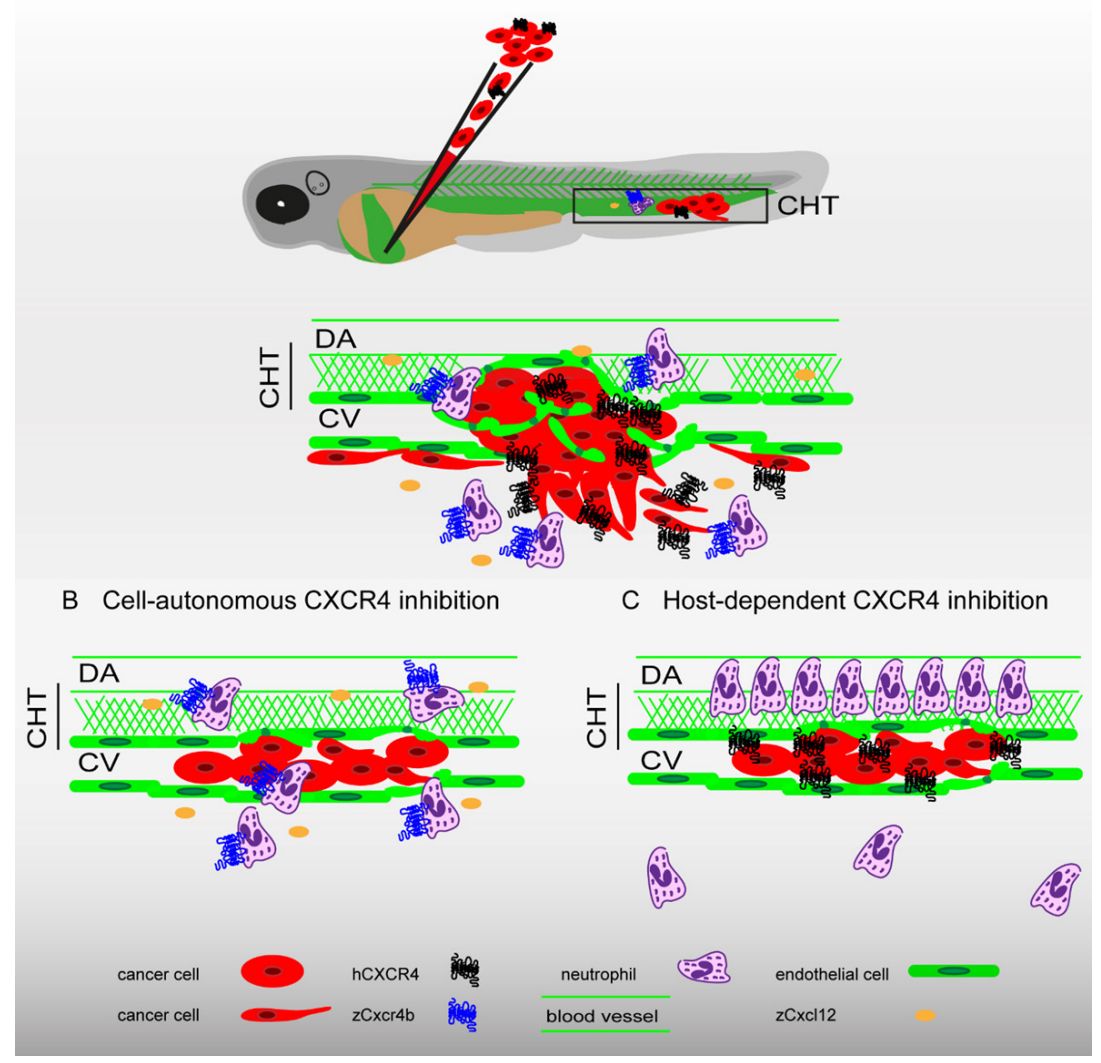

Figure 3. Role of cell-autonomous and host-dependent CXCR4 signalling in experimental metastasis formation in the zebrafish xenograft model. A: inoculation of human tumour cells into the blood circulation of zebrafish embryos results in experimental metastasis formation, characterized by tumour cell aggregates in the blood vessels, and extravasation and invasion in the surrounding tissue, in the region of the caudal hematopoietic tissue (CHT). During early metastatic events, endothelium alteration takes place and neutrophils localize in the surrounding of the tumour. The $\mathrm{CHT}$ is a vascular plexus in the tail fin between the DA and the CV and is a hematopoietic site; $\mathrm{B}$ : upon disruption of the tumour cell-autonomous CXCR4 signalling, cancer cells are unable to initiate early metastatic events, while surrounded by immune cells; $\mathrm{C}$ : the same inhibition of experimental metastasis formation occurs upon disruption of the host-dependent CXCR4 (Cxcr4b) signalling. Neutrophils are preferentially retained in the $\mathrm{CHT}$ and their recruitment at the metastatic site is impaired upon $\mathrm{Cxcr} 4$ signalling inhibition

We have previously shown that the impairment of the cell autonomous CXCR4 signalling blocks triplenegative breast cancer (TNBC) early metastatic events in the zebrafish xenograft model [Figure 3A and B]. In our model, human triple-negative breast cancer cells, derived from bone metastases developed in a mouse model, were implanted directly into the blood circulation of zebrafish embryos. Using this model, the formation of the primary tumour and the initial steps of metastasis (local invasion and intravasation into the blood circulation) were by-passed. Tumour cells, inoculated into the blood circulation, were found to form early metastases, by adhering to the endothelial wall, forming aggregates and invading the local tail fin tissue. Experimental metastases occurred in proximity of the caudal hematopoietic tissue, an intermediate site of hematopoiesis and a functional analogue of the fetal liver during mammalian development. This observation was in line with breast cancer metastasis formation in the bone ${ }^{[89,90]}$. In addition, others have also shown that tumour-derived CXCR4 signalling, in concert with the transcription factor Pit-1, drives tumour growth, in a zebrafish model ${ }^{[91,92]}$. Moreover, we demonstrated that the CXCR4 signalling functions across human and zebrafish systems, because CXCR4-expressing human cells respond to zebrafish Cxcl12 ligands and Cxcr4-expressing zebrafish cells migrate towards human CXCL12, showing that the zebrafish xenograft model is a valid approach to study human tumours. Taking advantage of the same in vivo model, where the interspecies crosstalk is validated, we propose a recently described CXCR4 antagonist, IT1t, as a possible therapeutic to inhibit early metastasis of $\mathrm{TNBC}^{[93]}$. In particular, breast 
cancer cells pre-treated in vitro with the CXCR4 antagonist ITit displayed reduced metastatic potential in zebrafish. Impaired tumour burden in vivo was also observed upon genetic inhibition of tumour-derived CXCR4 or microenvironment-dependent Cxcl12. In conclusion, we showed that the xenograft approach in zebrafish is a valuable model to study human tumours as the CXCR4 signalling functions in human cells upon zebrafish CXCL12 stimulation and vice versa CXCR4-expressing zebrafish cells respond to the human cognate chemokine.

\section{HOST-DEPENDENT CXCR4 SIGNALLING: CXCR4 CONTROLS THE TUMOUR METASTATIC NICHE PREPARATION, BY REGULATING INTRINSIC NEUTROPHIL FUNCTION AND RESPONSE TO CANCER CELLS}

Immune cells are programmed to recognise and eliminate transformed cells. However, cancer cells have evolved mechanisms that reprogram the immune defence and make the foe-to-friend switch an important support for survival and progression. The combination of chemotherapy and immunotherapy is a current strategy in the clinic ${ }^{[94]}$. Galluzzi et al. ${ }^{[95]}$ have recently reviewed anti-cancer therapies that re-activate the immune system, such as tumour-targeting antibodies, adoptive cell transfer and oncolytic viruses (all classified as passive immunotherapy), dendritic cell-based immunotherapies, anti-cancer vaccines, immune-stimulatory cytokines, immunomodulatory antibodies, inhibitors of immunosuppressive metabolism, pattern recognition receptor agonist, and immunogenic cell death inducers (all classified as active immunotherapy). Antibodies against CXCR4 are included in immunotherapeutic agents that skew the balance between M2/M1 TAMs toward the pro-inflammatory and anti-tumour M1 phenotype ${ }^{[5]}$.

We have recently shown the role of the host dependent CXCR4 signalling in supporting early metastatic events in the zebrafish xenograft model. Previous work from our group has shown that neutrophils are involved in the metastatic niche preparation by conditioning the ECM during their apparent random walk in the transmigration from the CHT (caudal hematopoietic tissue, transient hematopoietic site) to the tail tissue of zebrafish embryos ${ }^{[60]}$. Because CXCR4 is known to regulate the retention of hematopoietic stem progenitor cells (HSPCs) and differentiated leukocytes in the bone marrow in mammals ${ }^{[96]}$, and is highly expressed in zebrafish myeloid cells ${ }^{[97]}$, we hypothesised that CXCR4 signalling plays a role in controlling intrinsic neutrophil motility in physiological conditions. We found that neutrophils display altered motility and their number fluctuates during embryo development, leading to the conclusion that CXCR4 regulates neutrophil development in zebrafish. Moreover, a link between CXCR4 signalling and neutrophil response during inflammation has been recently described ${ }^{[98]}$. In our model, the neutrophilic response towards cancer cells was also altered in zebrafish mutants with a non-functional Cxcr4 (Cxcr4b). We identified a population of neutrophils that was mainly retained in the CHT and a population of neutrophils that even if moving in the tissue, displayed the inability to infiltrate tumour cell aggregates in the tail fin of Cxcr4b-null mutants. In the surrounding of cancer cells, cxcr4b-expressing neutrophils reduced their speed in motility, while Cxcr4b-null neutrophils maintained similar speeds as in neutrophils that had not been challenged by cancer cells. Therefore, we propose that Cxcr4 controls neutrophil development and response to tumour cells, initiating early metastatic events [Figure $3 \mathrm{~A}$ and C]. RNA sequencing performed on sorted neutrophils from wild-type or $c x c r 4 b-/-$ zebrafish larvae supported our conclusion that motility and adhesion are altered when neutrophils lack a functional Cxcr4 signalling ${ }^{[99]}$. In conclusion, we propose that these alterations are responsible for the impaired tumour niche preparation and inhibition of early micrometastasis formation in different types of cancer.

\section{CONCLUSION}

Cancer is a complex, multi-step disease and the second leading cause of death worldwide [ 1 in 6 deaths is due to cancer, 9.6 million cancer-related deaths in 2018 (www.who.int, October 2019)]. Patients diagnosed 
with primary tumours are treated, when possible, with surgery. However, metastasis can occur years after surgical intervention ${ }^{[100]}$. Metastatic cancer associates with poor patient prognosis and represent a major challenge for clinical research. Chemotherapy is often the pharmacological choice to treat cancer, although side effects alter normal cell physiology and affect patient life quality. Moreover, cancer relapse and therapy resistance associate with poor prognosis. Progress in biomedical research has shown that targeting cancer cells is not the only therapeutic option. The interaction between tumour and surrounding stroma supports cancer survival and spreading, representing therefore a possible new treatment strategy ${ }^{[101]}$. Here, we describe the use of the zebrafish xenograft model to study early stages of experimental micrometastasis formation, engrafting fluorescent tumour cells in transparent zebrafish embryos with fluorescent endothelial and immune cells. We propose that targeting CXCR4 signalling on cancer cells or in the tumour microenvironment is a valid approach to inhibit metastatic cancer and suggest that antiCXCR4 therapy might have double treatment benefits. In addition, therapeutic modulation of the immune system might result in the reinforcement of the immune defence against cancer. However, we suggest that treatments designed to target malignant cells might affect tumour microenvironment intrinsic functions. Specifically, the intrinsic physiological role of myeloid cells can be affected by cancer treatment, resulting in an inability to mount a functional anti-cancer response or, on the other hand, in the ability to mount a tumour-supportive response.

\section{DECLARATIONS}

\section{Authors' contributions}

Wrote and reviewed the manuscript: Tulotta C, Snaar-Jagalska BE

\section{Availability of data and materials}

Not applicable.

\section{Financial support and sponsorship}

The work was supported by the Netherlands Organization for Scientific Research (TOP GO Grant: 854.10.012).

\section{Conflicts of interest}

Both authors declared that there are no conflicts of interest.

\section{Ethical approval and consent to participate}

Not applicable.

\section{Consent for publication}

Not applicable.

\section{Copyright}

(c) The Author(s) 2019.

\section{REFERENCES}

1. Hanahan D, Weinberg RA. Hallmarks of cancer: the next generation. Cell 2011;144:646-74.

2. Klemm F, Joyce JA. Microenvironmental regulation of therapeutic response in cancer. Trends Cell Biol 2015;25:198-213.

3. Granot Z, Fridlender ZG. Plasticity beyond cancer cells and the "Immunosuppressive Switch". Cancer Res 2015;75:4441-5.

4. Coffelt SB, Kersten K, Doornebal CW, Weiden J, Vrijland K, et al. IL-17-producing gammadelta T cells and neutrophils conspire to promote breast cancer metastasis. Nature 2015;522:345-8.

5. Wang J, Knaut H. Chemokine signaling in development and disease. Development 2014;141:4199-205.

6. Zlotnik A, Yoshie O. The chemokine superfamily revisited. Immunity 2012;36:705-16. 
7. Karnoub AE, Weinberg RA. Chemokine networks and breast cancer metastasis. Breast Dis 2006;26:75-85.

8. Kiefer F, Siekmann AF. The role of chemokines and their receptors in angiogenesis. Cell Mol Life Sci 2011;68:2811-30.

9. Boldajipour B, Mahabaleshwar H, Kardash E, Reichman-Fried M, Blaser H, et al. Control of chemokine-guided cell migration by ligand sequestration. Cell 2008;132:463-73.

10. Dona E, Barry JD, Valentin G, Quirin C, Khmelinskii A, et al. Directional tissue migration through a self-generated chemokine gradient. Nature 2013;503:285-9.

11. Venkiteswaran G, Lewellis SW, Wang J, Reynolds E, Nicholson C, et al. Generation and dynamics of an endogenous, self-generated signaling gradient across a migrating tissue. Cell 2013;155:674-87.

12. Pawig L, Klasen C, Weber C, Bernhagen J, Noels H. Diversity and inter-connections in the CXCR4 chemokine receptor/ligand family: molecular perspectives. Front Immunol 2015;6:429.

13. Scala S. Molecular pathways: targeting the CXCR4-CXCL12 axis-untapped potential in the tumor microenvironment. Clin Cancer Res 2015;21:4278-85.

14. Nguyen DX, Bos PD, Massague J. Metastasis: from dissemination to organ-specific colonization. Nat Rev Cancer 2009;9:274-84.

15. Xue LJ, Mao XB, Ren LL, Chu XY. Inhibition of CXCL12/CXCR4 axis as a potential targeted therapy of advanced gastric carcinoma. Cancer Med 2017;6:1424-36.

16. Mitchell B, Mahalingam M. The CXCR4/CXCL12 axis in cutaneous malignancies with an emphasis on melanoma. Histol Histopathol 2014;29:1539-46.

17. Xu CZ, Wang PH, Yan XJ, Wang T, Chen D, et al. Expression of CXCR4 is associated with progression and invasion in patients with nasal-surface basal cell carcinoma. ORL J Otorhinolaryngol Relat Spec 2013;75:332-41.

18. Knopf A, Bahadori L, Fritsche K, Piontek G, Becker CC, et al. Primary tumor-associated expression of CXCR4 predicts formation of local and systemic recurrency in head and neck squamous cell carcinoma. Oncotarget 2017;8:112739-47.

19. Wald O. CXCR4 based therapeutics for non-small cell lung cancer (NSCLC). J Clin Med 2018;7:E303.

20. Xu C, Zhao H, Chen H, Yao Q. CXCR4 in breast cancer: oncogenic role and therapeutic targeting. Drug Des Devel Ther 2015;9:4953-64.

21. Figueras A, Alsina-Sanchis E, Lahiguera A, Abreu M, Muinelo-Romay L, et al. A role for CXCR4 in peritoneal and hematogenous ovarian cancer dissemination. Mol Cancer Ther 2018;17:532-43.

22. Bao Y, Wang Z, Liu B, Lu X, Xiong Y, et al. A feed-forward loop between nuclear translocation of CXCR4 and HIF-1alpha promotes renal cell carcinoma metastasis. Oncogene 2019;38:881-95.

23. Zhu WB, Zhao ZF, Zhou X. AMD3100 inhibits epithelial-mesenchymal transition, cell invasion, and metastasis in the liver and the lung through blocking the SDF-1alpha/CXCR4 signaling pathway in prostate cancer. J Cell Physiol 2019;234:11746-59.

24. Richardson PJ. CXCR4 and glioblastoma. Anticancer Agents Med Chem 2016;16:59-74.

25. Sand LG, Scotlandi K, Berghuis D, Snaar-Jagalska BE, Picci P, et al. CXCL14, CXCR7 expression and CXCR4 splice variant ratio associate with survival and metastases in Ewing sarcoma patients. Eur J Cancer 2015;51:2624-33.

26. Du W, Lu C, Zhu X, Hu D, Chen X, et al. Prognostic significance of CXCR4 expression in acute myeloid leukemia. Cancer Med 2019; doi: $10.1002 /$ cam4.2535.

27. Choi WT, Yang Y, Xu Y, An J. Targeting chemokine receptor CXCR4 for treatment of HIV-1 infection, tumor progression, and metastasis. Curr Top Med Chem 2014;14:1574-89.

28. Guo F, Wang Y, Liu J, Mok SC, Xue F, et al. CXCL12/CXCR4: a symbiotic bridge linking cancer cells and their stromal neighbors in oncogenic communication networks. Oncogene 2016;35:816-26.

29. Sugihara H, Ishimoto T, Yasuda T, Izumi D, Eto K, et al. Cancer-associated fibroblast-derived CXCL12 causes tumor progression in adenocarcinoma of the esophagogastric junction. Med Oncol 2015;32:618.

30. Fearon DT. The carcinoma-associated fibroblast expressing fibroblast activation protein and escape from immune surveillance. Cancer Immunol Res 2014;2:187-93.

31. Feig C, Jones JO, Kraman M, Wells RJ, Deonarine A, et al. Targeting CXCL12 from FAP-expressing carcinoma-associated fibroblasts synergizes with anti-PD-L1 immunotherapy in pancreatic cancer. Proc Natl Acad Sci U S A 2013;110:20212-7.

32. Brandau S, Trellakis S, Bruderek K, Schmaltz D, Steller G, et al. Myeloid-derived suppressor cells in the peripheral blood of cancer patients contain a subset of immature neutrophils with impaired migratory properties. J Leukoc Biol 2011;89:311-7.

33. Contento RL, Molon B, Boularan C, Pozzan T, Manes S, et al. CXCR4-CCR5: a couple modulating T cell functions. Proc Natl Acad Sci U S A 2008;105:10101-6.

34. Strydom N, Rankin SM. Regulation of circulating neutrophil numbers under homeostasis and in disease. J Innate Immun 2013;5:304-14.

35. Zou L, Barnett B, Safah H, Larussa VF, Evdemon-Hogan M, et al. Bone marrow is a reservoir for CD4+CD25+ regulatory T cells that traffic through CXCL12/CXCR4 signals. Cancer Res 2004;64:8451-5.

36. Jablonska J, Leschner S, Westphal K, Lienenklaus S, Weiss S. Neutrophils responsive to endogenous IFN-beta regulate tumor angiogenesis and growth in a mouse tumor model. J Clin Invest 2010;120:1151-64.

37. Hughes R, Qian BZ, Rowan C, Muthana M, Keklikoglou I, et al. Perivascular M2 macrophages stimulate tumor relapse after chemotherapy. Cancer Res 2015;75:3479-91.

38. Ping YF, Yao XH, Jiang JY, Zhao LT, Yu SC, et al. The chemokine CXCL12 and its receptor CXCR4 promote glioma stem cellmediated VEGF production and tumour angiogenesis via PI3K/AKT signalling. J Pathol 2011;224:344-54.

39. Beider K, Bitner H, Leiba M, Gutwein O, Koren-Michowitz M, et al. Multiple myeloma cells recruit tumor-supportive macrophages through the CXCR4/CXCL12 axis and promote their polarization toward the M2 phenotype. Oncotarget 2014;5:11283-96.

40. Gil M, Komorowski MP, Seshadri M, Rokita H, McGray AJ, et al. CXCL12/CXCR4 blockade by oncolytic virotherapy inhibits 
ovarian cancer growth by decreasing immunosuppression and targeting cancer-initiating cells. J Immunol 2014;193:5327-37.

41. Guo ZS. The 2018 Nobel Prize in medicine goes to cancer immunotherapy (editorial for BMC cancer). BMC Cancer 2018;18:1086.

42. Chen IX, Chauhan VP, Posada J, Ng MR, Wu MW, et al. Blocking CXCR4 alleviates desmoplasia, increases T-lymphocyte infiltration, and improves immunotherapy in metastatic breast cancer. Proc Natl Acad Sci U S A 2019;116:4558-66.

43. Patton EE, Dhillon P, Amatruda JF, Ramakrishnan L. Spotlight on zebrafish: translational impact. Dis Model Mech 2014;7:731-3.

44. Antonio N, Bonnelykke-Behrndtz ML, Ward LC, Collin J, Christensen IJ, et al. The wound inflammatory response exacerbates growth of pre-neoplastic cells and progression to cancer. EMBO J 2015;34:2219-36.

45. Feng Y, Renshaw S, Martin P. Live imaging of tumor initiation in zebrafish larvae reveals a trophic role for leukocyte-derived PGE(2). Curr Biol 2012;22:1253-9.

46. Feng Y, Santoriello C, Mione M, Hurlstone A, Martin P. Live imaging of innate immune cell sensing of transformed cells in zebrafish larvae: parallels between tumor initiation and wound inflammation. PLoS Biol 2010;8:e1000562.

47. Kimmel CB, Ballard WW, Kimmel SR, Ullmann B, Schilling TF. Stages of embryonic development of the zebrafish. Dev Dyn 1995;203:253-310.

48. Ellett F, Pase L, Hayman JW, Andrianopoulos A, Lieschke GJ. mpeg1 promoter transgenes direct macrophage-lineage expression in zebrafish. Blood 2011;117:e49-56.

49. Lawson ND, Weinstein BM. In vivo imaging of embryonic vascular development using transgenic zebrafish. Dev Biol 2002;248:307-18.

50. Renshaw SA, Loynes CA, Trushell DM, Elworthy S, Ingham PW, et al. A transgenic zebrafish model of neutrophilic inflammation. Blood 2006;108:3976-8.

51. Gonzales AP, Yeh JR. Cas9-based genome editing in zebrafish. Methods Enzymol 2014;546:377-413.

52. Lawson ND, Wolfe SA. Forward and reverse genetic approaches for the analysis of vertebrate development in the zebrafish. Dev Cell 2011;21:48-64.

53. Robertson AL, Holmes GR, Bojarczuk AN, Burgon J, Loynes CA, et al. A zebrafish compound screen reveals modulation of neutrophil reverse migration as an anti-inflammatory mechanism. Sci Transl Med 2014;6:225ra29.

54. Goessling W, North TE, Zon LI. New waves of discovery: modeling cancer in zebrafish. J Clin Oncol 2007;25:2473-9.

55. Ung CY, Lam SH, Gong Z. Comparative transcriptome analyses revealed conserved biological and transcription factor target modules between the zebrafish and human tumors. Zebrafish 2009;6:425-31.

56. Amatruda JF, Shepard JL, Stern HM, Zon LI. Zebrafish as a cancer model system. Cancer Cell 2002;1:229-31.

57. Letrado P, de Miguel I, Lamberto I, Diez-Martinez R, Oyarzabal J. Zebrafish: speeding up the cancer drug discovery process. Cancer Res 2018;78:6048-58.

58. van der Ent W, Veneman WJ, Groenewoud A, Chen L, Tulotta C, et al. Automation of technology for cancer research. Adv Exp Med Biol 2016;916:315-32.

59. Zon LI, Peterson RT. In vivo drug discovery in the zebrafish. Nat Rev Drug Discov 2005;4:35-44.

60. He SN, Lamers GEM, Beenakker JWM, Cui C, Ghotra VPS, et al. Neutrophil-mediated experimental metastasis is enhanced by VEGFR inhibition in a zebrafish xenograft model. J Pathol 2012;227:431-45.

61. Tulotta C, He S, Chen L, Groenewoud A, van der Ent W, et al. Imaging of human cancer cell proliferation, invasion, and micrometastasis in a Zebrafish xenogeneic engraftment model. Methods Mol Biol 2016;1451:155-69.

62. Tulotta C, He S, van der Ent W, Chen L, Groenewoud A, et al. Imaging cancer angiogenesis and metastasis in a Zebrafish embryo model. Adv Exp Med Biol 2016;916:239-63.

63. Astone M, Dankert EN, Alam SK, Hoeppner LH. Fishing for cures: the alLURE of using zebrafish to develop precision oncology therapies. NPJ Precis Oncol 2017;1:39.

64. Gaudenzi G, Albertelli M, Dicitore A, Wurth R, Gatto F, et al. Patient-derived xenograft in zebrafish embryos: a new platform for translational research in neuroendocrine tumors. Endocrine 2017;57:214-9.

65. Mercatali L, La Manna F, Groenewoud A, Casadei R, Recine F, et al. Development of a patient-derived xenograft (PDX) of breast cancer bone metastasis in a Zebrafish model. Int J Mol Sci 2016;17:E1375.

66. Wu JQ, Zhai J, Li CY, Tan AM, Wei P, et al. Patient-derived xenograft in zebrafish embryos: a new platform for translational research in gastric cancer. J Exp Clin Cancer Res 2017;36:160.

67. Tulotta C, Groenewoud A, Snaar-Jagalska BE, Ottewell P. Animal models of breast cancer bone metastasis. Methods Mol Biol 2019;1914:309-30.

68. Vazquez Rodriguez G, Abrahamsson A, Jensen LD, Dabrosin C. Estradiol promotes breast cancer cell migration via recruitment and activation of neutrophils. Cancer Immunol Res 2017;5:234-47.

69. Kiener M, Chen L, Krebs M, Grosjean J, Klima I, et al. miR-221-5p regulates proliferation and migration in human prostate cancer cells and reduces tumor growth in vivo. BMC Cancer 2019;19:627.

70. Ghotra VP, He S, van der Horst G, Nijhoff S, de Bont H, et al. SYK is a candidate kinase target for the treatment of advanced prostate cancer. Cancer Res 2015;75:230-40.

71. Canella A, Welker AM, Yoo JY, Xu J, Abas FS, et al. Efficacy of Onalespib, a long-acting second-generation HSP90 inhibitor, as a single agent and in combination with temozolomide against malignant gliomas. Clin Cancer Res 2017;23:6215-26.

72. Vandercappellen J, Van Damme J, Struyf S. The role of CXC chemokines and their receptors in cancer. Cancer Lett 2008;267:226-44.

73. Nagasawa T, Hirota S, Tachibana K, Takakura N, Nishikawa S, et al. Defects of B-cell lymphopoiesis and bone-marrow myelopoiesis in mice lacking the CXC chemokine PBSF/SDF-1. Nature 1996;382:635-8.

74. Rosu-Myles M, Gallacher L, Murdoch B, Hess DA, Keeney M, et al. The human hematopoietic stem cell compartment is 
heterogeneous for CXCR4 expression. Proc Natl Acad Sci U S A 2000;97:14626-31.

75. Day RB, Link DC. Regulation of neutrophil trafficking from the bone marrow. Cell Mol Life Sci 2012;69:1415-23.

76. Sallusto F, Baggiolini M. Chemokines and leukocyte traffic. Nat Immunol 2008;9:949-52.

77. Sallusto F, Mackay CR, Lanzavecchia A. The role of chemokine receptors in primary, effector, and memory immune responses. Annu Rev Immunol 2000;18:593-620.

78. Bussmann J, Raz E. Chemokine-guided cell migration and motility in zebrafish development. EMBO J 2015;34:1309-18.

79. Vicenzi E, Lio P, Poli G. The puzzling role of CXCR4 in human immunodeficiency virus infection. Theranostics 2013;3:18-25.

80. Gulino AV. WHIM syndrome: a genetic disorder of leukocyte trafficking. Curr Opin Allergy Clin Immunol 2003;3:443-50.

81. Balkwill F. The significance of cancer cell expression of the chemokine receptor CXCR4. Semin Cancer Biol 2004;14:171-9.

82. Chatterjee S, Behnam Azad B, Nimmagadda S. The intricate role of CXCR4 in cancer. Adv Cancer Res 2014;124:31-82.

83. Saini V, Marchese A, Majetschak M. CXC chemokine receptor 4 is a cell surface receptor for extracellular ubiquitin. J Biol Chem 2010;285:15566-76.

84. Bernhagen J, Krohn R, Lue H, Gregory JL, Zernecke A, et al. MIF is a noncognate ligand of CXC chemokine receptors in inflammatory and atherogenic cell recruitment. Nat Med 2007;13:587-96.

85. Lourenco S, Teixeira VH, Kalber T, Jose RJ, Floto RA, et al. Macrophage migration inhibitory factor-CXCR4 is the dominant chemotactic axis in human mesenchymal stem cell recruitment to tumors. J Immunol 2015;194:3463-74.

86. Shin HN, Moon HH, Ku JL. Stromal cell-derived factor-1alpha and macrophage migration-inhibitory factor induce metastatic behavior in CXCR4-expressing colon cancer cells. Int J Mol Med 2012;30:1537-43.

87. Lu J, Chatterjee M, Schmid H, Beck S, Gawaz M. CXCL14 as an emerging immune and inflammatory modulator. J Inflamm (Lond) 2016;13:1.

88. Muller A, Homey B, Soto H, Ge N, Catron D, et al. Involvement of chemokine receptors in breast cancer metastasis. Nature 2001;410:50-6.

89. Weidle UH, Birzele F, Kollmorgen G, Ruger R. Molecular mechanisms of bone metastasis. Cancer Genomics Proteomics 2016;13:1-12.

90. Ottewell PD, O'Donnell L, Holen I. Molecular alterations that drive breast cancer metastasis to bone. Bonekey Rep 2015;4:643.

91. Martinez-Ordonez A, Seoane S, Cabezas P, Eiro N, Sendon-Lago J, et al. Breast cancer metastasis to liver and lung is facilitated by Pit-1-CXCL12-CXCR4 axis. Oncogene 2018;37:1430-44.

92. Seoane S, Martinez-Ordonez A, Eiro N, Cabezas-Sainz P, Garcia-Caballero L, et al. POU1F1 transcription factor promotes breast cancer metastasis via recruitment and polarization of macrophages. J Pathol 2019;249:381-94.

93. Tulotta C, Stefanescu C, Beletkaia E, Bussmann J, Tarbashevich K, et al. Inhibition of signaling between human CXCR4 and zebrafish ligands by the small molecule IT1t impairs the formation of triple-negative breast cancer early metastases in a zebrafish xenograft model. Dis Model Mech 2016;9:141-53.

94. Cook AM, Lesterhuis WJ, Nowak AK, Lake RA. Chemotherapy and immunotherapy: mapping the road ahead. Curr Opin Immunol 2016;39:23-9.

95. Galluzzi L, Vacchelli E, Bravo-San Pedro JM, Buque A, Senovilla L, et al. Classification of current anticancer immunotherapies. Oncotarget 2014;5:12472-508.

96. Tchernychev B, Ren Y, Sachdev P, Janz JM, Haggis L, et al. Discovery of a CXCR4 agonist pepducin that mobilizes bone marrow hematopoietic cells. Proc Natl Acad Sci U S A 2010;107:22255-9.

97. Walters KB, Green JM, Surfus JC, Yoo SK, Huttenlocher A. Live imaging of neutrophil motility in a zebrafish model of WHIM syndrome. Blood 2010;116:2803-11.

98. Isles HM, Herman KD, Robertson AL, Loynes CA, Prince LR, et al. The CXCL12/CXCR4 signaling axis retains neutrophils at inflammatory sites in Zebrafish. Front Immunol 2019;10:1784.

99. Tulotta C, Stefanescu C, Chen Q, Torraca V, Meijer AH, et al. CXCR4 signaling regulates metastatic onset by controlling neutrophil motility and response to malignant cells. Sci Rep 2019;9:2399.

100. Demicheli R, Retsky MW, Hrushesky WJ, Baum M, Gukas ID. The effects of surgery on tumor growth: a century of investigations. Ann Oncol 2008;19:1821-8

101. Sounni NE, Noel A. Targeting the tumor microenvironment for cancer therapy. Clin Chem 2013;59:85-93. 